\title{
Holographic interferometry under phase distortions
}

\author{
L.A. Derzhypolska, O.V. Gnatovskiy \\ Institute of Physics, NAS of Ukraine, 46, prospect Nauky, 03028 Kyiv, Ukraine \\ Phone: +38 (044) 525-99-68
}

\begin{abstract}
An impact of non-stationary phase distortions in transfer section of a holographic interferometer on the quality of formed interference fringes in an interferogram was under investigation. A mathematical model to analyze this influence was proposed. The conclusions based on the model were made about the possibility to observe interference fringes under phase noise in the broad ranges. Theoretical estimations were involved to analyze the experimental data. For that purpose, a modified holographic interferometer scheme with regular bundle of multimode lightguiding fibers in the transfer section was used. Investigated here was the possibility to use the scheme under possible deformations of the bundle between the two interferometric exposures. Experimentally proved was the capability of the holographic interferometer with flexible lightguiding bundle to operate under reasonable deformations of the bundle and even under non-rigid fixation of bundle body.
\end{abstract}

Keywords: holographic interferometry, regular lightguiding fiber bundle, phase distortions.

Manuscript received 17.03.06; accepted for publication 23.10.06.

\section{Introduction}

The essence of holographic interferometry is a comparison of the wave-fronts of an investigated object in different states. In this case, the quality of the wavefronts of the object is supposed to remain unchangeable during the investigations. However, besides the changes of the state of the object, which are the subject of investigation, there are possible an unexpected wavefront modifications due to atmosphere fluctuations, a dust deposition over the object, distortions in imagetransferring systems, etc. Negative factors will impair interference fringes that may have effect on accuracy of measurements. Therefore, it seems that the influence of the distortions needs to be ascertained. Such an investigation was a subject of this work.

\section{Theory}

The proposed consideration is based on the following model. As shown in Fig. 1, a laser radiation in an initial plane $\left(x_{1}, y_{1}\right)$ is scattered by the surface of the investigated object $A=a\left(x_{1}, y_{1}\right) \exp \left[i \varphi\left(x_{1}, y_{1}\right)\right] \quad$ in two different states. Assume for determinacy that in the second state the object is turned through a small angle $\alpha$, and its scattered wave-front takes a phase addition $\Delta \varphi=\exp [i(\alpha x)]$. The images of the two states of object are being transferred by the optical system $\mathrm{O}_{1} \ldots \mathrm{O}_{2}$ through some distorting media DM to the image plane $\left(x_{2}, y_{2}\right)$, recording two holograms in the registering element $\mathrm{H}$ (see Fig. 1). Phase distortion for the first exposure appears as an additional phase $\psi_{1}\left(x_{2}, y_{2}\right)$, and $\psi_{2}\left(x_{2}, y_{2}\right)$ - for the second one. Under illumination of the recorded hologram an interference field is formed in the plane $\left(x_{2}, y_{2}\right)$ with an intensity $I \sim \mid A_{1}\left(x_{2}, y_{2}\right)+$ $+\left.A_{2}\left(x_{2}, y_{2}\right)\right|^{2}$.

Denote $\psi_{1}\left(x_{2}, y_{2}\right)-\psi_{2}\left(x_{2}, y_{2}\right)=\psi\left(x_{2}, y_{2}\right)$ and obtain:

$$
\begin{aligned}
& I \sim 2|a|^{2}+|a|^{2} \exp \left\{i\left[\psi\left(x_{2}, y_{2}\right)-\alpha_{2} x_{2}\right]\right\}+ \\
& +|a|^{2} \exp \left\{-i\left[\psi\left(x_{2}, y_{2}\right)-\alpha_{2} x_{2}\right]\right\}= \\
& =2|a|^{2}\left\{1+\cos \left[\alpha x_{2}-\psi\left(x_{2}, y_{2}\right)\right]\right\} .
\end{aligned}
$$

This expression describes object luminosity $\left|a\left(x_{2}, y_{2}\right)\right|^{2}$ modulated with an interference pattern $\{1+$ $\left.\cos \left[\alpha x_{2}-\psi\left(x_{2}, y_{2}\right)\right]\right\}$. Now let's analyze the result.

If there is no distorting medium or it is stationary then $\psi=0, I_{\max }=2, I_{\min }=0$ and the fringe contrast defined as $V=\frac{I_{\max }-I_{\min }}{I_{\max }+I_{\min }}[1] \quad$ takes a value of 1 according to the known estimation [2]. In the case of non-stationary phase noise $\psi \neq 0$. Generally it is a stochastic function. Let's find an average of the distribution $I(\psi)=\cos (\alpha x-\psi)$ defined as [3]: 


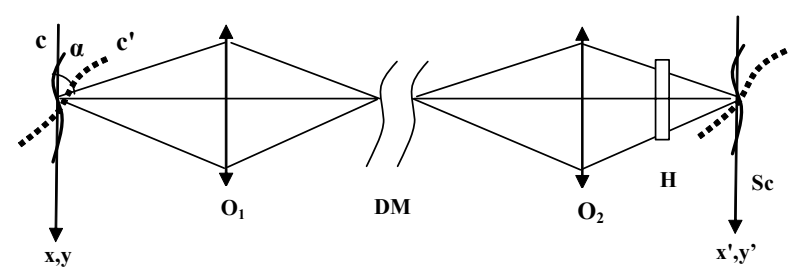

Fig. 1. Scheme of image transfer.

$\left\langle\cos \left(\alpha x^{\prime}-\psi\right)\right\rangle=\int_{-\infty}^{+\infty} \rho(\psi) \cos \left(\alpha x^{\prime}-\psi\right) d \psi=$

$\operatorname{Re} \int_{-\infty}^{+\infty} \rho(\psi) \exp \left[i\left(\alpha x^{\prime}-\psi\right)\right] d \psi=$

$=\operatorname{Re}\left[\exp [i \alpha x] \int_{-\infty}^{+\infty} \rho(\psi) \exp [i \psi] d \psi\right]$.

To evaluate the expression (2), we need to determine the distribution density $\rho(\psi)$. It's naturally to assume that the phase noise $\psi$ has the normal distribution density. Assume also that the average of $\psi$ equals to 0 , then the mean-square value $\left\langle\psi^{2}\right\rangle$ defines a standard deviation $\sigma^{2}$, and

$\rho(\psi)=\frac{1}{\sigma \sqrt{2 \pi}} \exp \left(-\frac{\psi^{2}}{2 \sigma^{2}}\right)$.

Substitute (3) into (2):

$\left\langle\cos \left(\alpha x^{\prime}-\psi\right)\right\rangle=$

$=\frac{1}{\sigma \sqrt{2 \pi}} \operatorname{Re}\left[\exp [i \alpha x] \int_{-\infty}^{+\infty} \exp \left(-\frac{\psi^{2}}{2 \sigma^{2}}\right) \exp [i \psi] d \psi\right]$.

As shown in [4]:

$\int_{-\infty}^{+\infty} \exp \left(i \psi-\frac{\psi^{2}}{2 \sigma^{2}}\right) d \psi=\sigma \sqrt{2 \pi} \exp \left(-\frac{\sigma^{2}}{2}\right)$.

In that way, the average of $\cos \left(\alpha x^{\prime}-\psi\right)$ is obtained:

$$
\begin{aligned}
& \left\langle\cos \left(\alpha x^{\prime}-\psi\right)\right\rangle=\frac{\sigma \sqrt{2 \pi}}{\sigma \sqrt{2 \pi}} \cos \left(\alpha x^{\prime}\right) \exp \left(-\frac{\sigma^{2}}{2}\right)= \\
& =\cos \left(\alpha x^{\prime}\right) \exp \left(-\frac{\sigma^{2}}{2}\right) .
\end{aligned}
$$

Let's find the maximum and the minimum of the averaged interference field intensity. As follows from (6), the maximum occurs when $\cos \left(\alpha x^{\prime}\right)=+1$, and the minimum occurs when $\cos \left(\alpha x^{\prime}\right)=-1$. Finally, for the fringe contrast under a phase noise obtain:

$$
\begin{aligned}
& I_{\max }=1+\exp \left(-\frac{\sigma^{2}}{2}\right) \\
& I_{\min }=1-\exp \left(-\frac{\sigma^{2}}{2}\right) \\
& \Rightarrow V^{\prime}=\frac{1+\exp \left(-\frac{\sigma^{2}}{2}\right)-1+\exp \left(-\frac{\sigma^{2}}{2}\right)}{1+\exp \left(-\frac{\sigma^{2}}{2}\right)+1-\exp \left(-\frac{\sigma^{2}}{2}\right)}=\exp \left(-\frac{\sigma^{2}}{2}\right)
\end{aligned}
$$

A plot of fringe contrast against mean-square deviation $V^{\prime}(\sigma)$ is shown at the left of Fig. 2. At the right of Fig. 1, a series of computer simulated images of the interference pattern for certain points on the plot are depicted. Fig. 3a shows several plots of density distribution functions for different $\sigma$. Being built on the basis of Fig. 3a, the plots of distribution functions $F\left(|\psi| \leq \psi_{0}\right)=\int_{-\psi_{0}}^{\psi_{0}} \rho(\psi) d \psi$ demonstrates the histograms for the same values of $\sigma$ that allow to estimate a relative contribution of phase noise of different amplitudes to the fringe contrast.

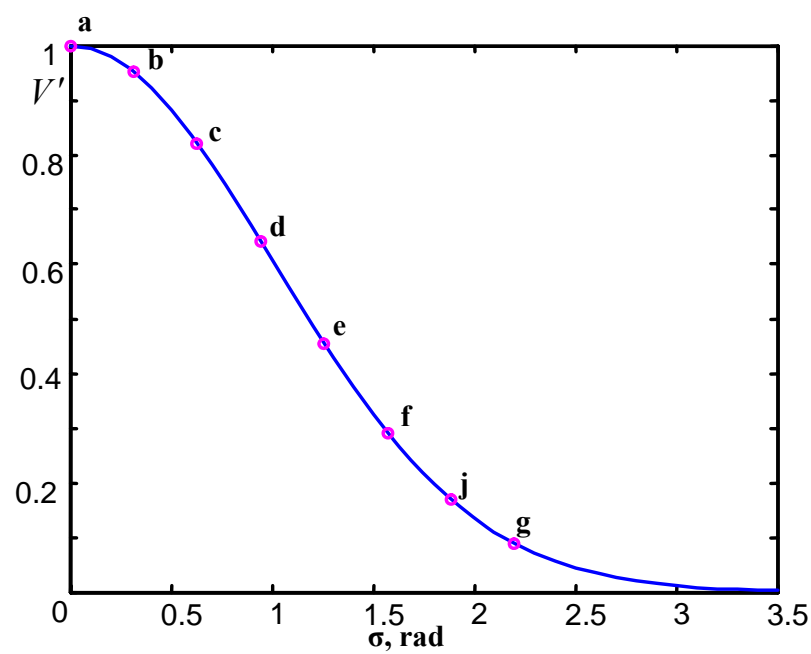

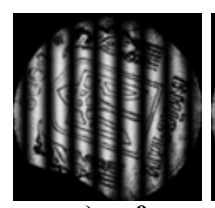

a) $\sigma=0$

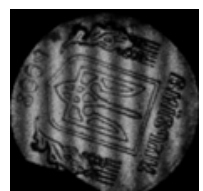

e) $\sigma=0.4 \pi$

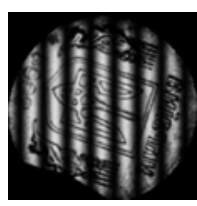

b) $\sigma=0.1 \pi$

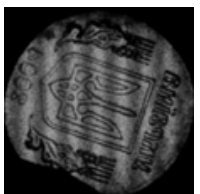

f) $\sigma=0.5 \pi$

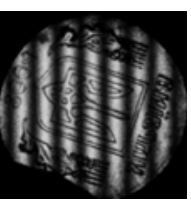

c) $\sigma=0.2 \pi$

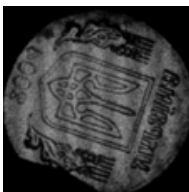

j) $\sigma=0.6 \pi$
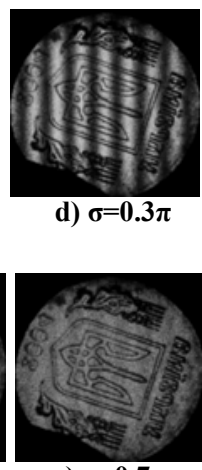

g) $\sigma=0.7 \pi$
Fig. 2. Plot of fringe contrast $V^{\prime}$ against $\sigma$ and views of interferograms for certain values of $\sigma$. 


\section{Experiment}

We invoked the results of theoretical consideration to analyze experimental data. A modified holographic interferometer scheme was used in the experiment with multimode lightguiding fiber bundle as a distorting media. A possibility to use the lightguiding fiber bundle for holographic information transfer was demonstrated in [5]. In this work, we investigated a capability of the scheme under possible deformations of the bundle between the two exposures of hologram for the comparable object states. In this case, in the second state

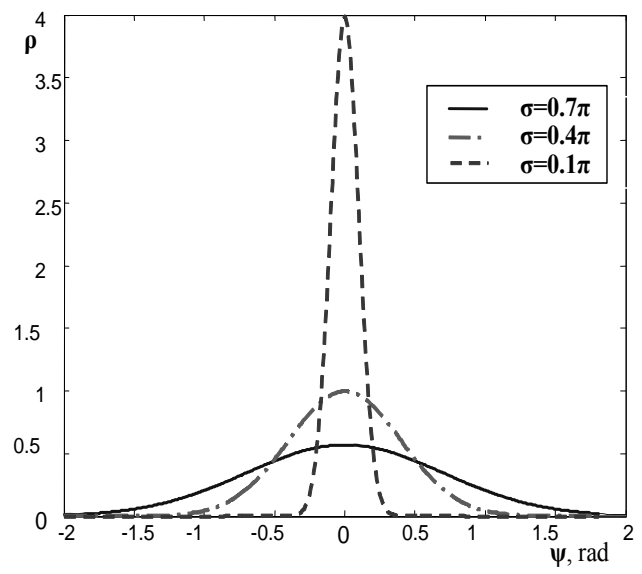

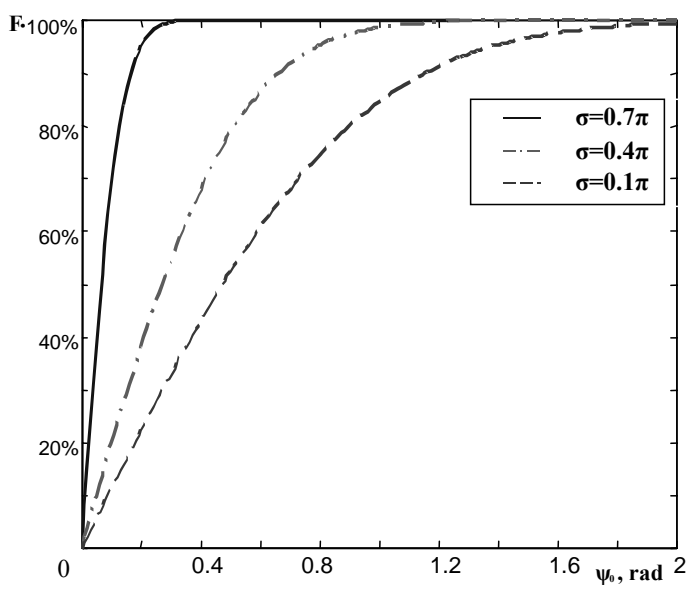

Fig. 3. a) Plot of the density of distribution $\rho(\psi)$ for several values of $\sigma$; b) the plot of distribution of the relative number of fibers on the magnitude of phase distortion $F\left(|\psi| \leq \psi_{0}\right)$.

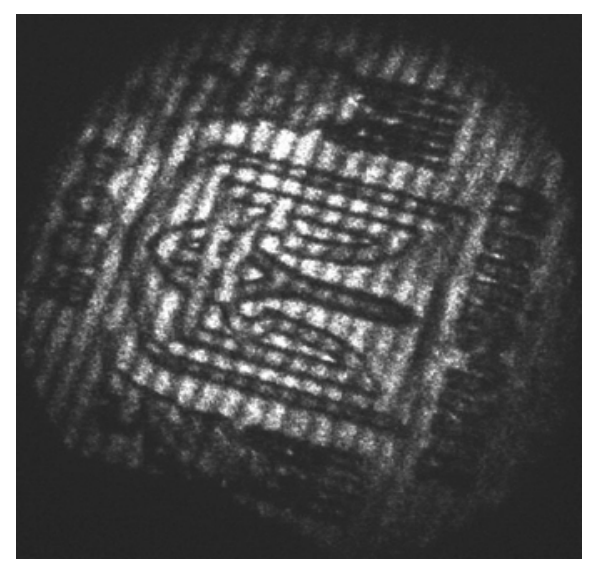

a)

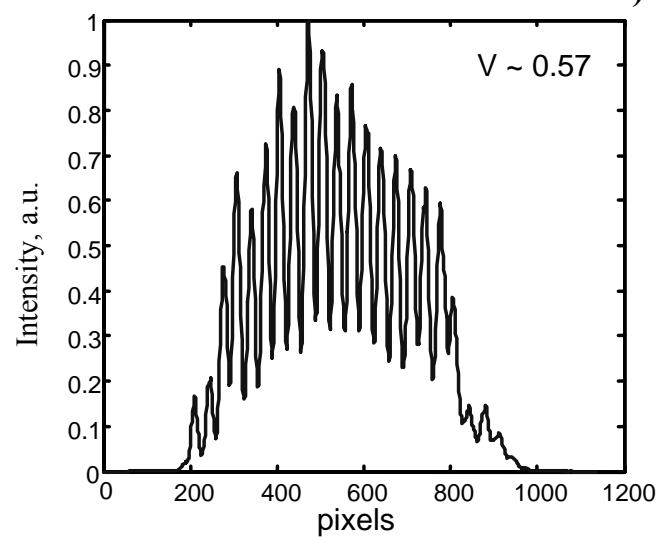

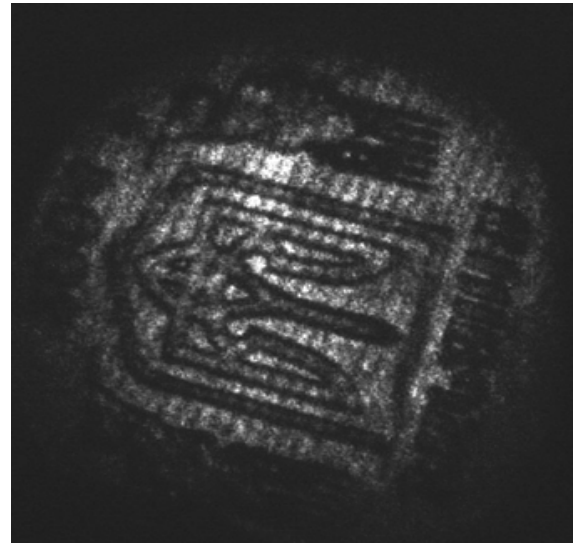

b)

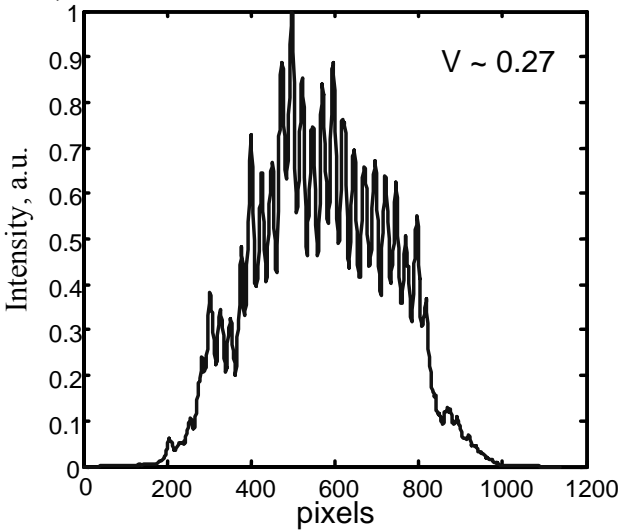

Fig. 4. a) Photograph of the interferogram of the object and the normalized plot of the intensity averaged along the fringes, a case of the undisturbed bundle; b) the same, a case of the disturbed bundle. 
the object is turned through a small angle $\alpha$, as considered in the theory. The image of the object is projected by $\mathrm{O}_{1}$ from the object onto the inlet bundle face and then from the outlet bundle face through the hologram onto the observing screen $\mathrm{Sc}$ by $\mathrm{O}_{2}$. Both ends of the bundle were clamped in the holders. Thereby the phase noise generator was managed on the random phase changes in the single fibers under the deformations of the bundle. Bending deflection of the bundle was reached as much as $7 \mathrm{~cm}$, while the bundle length was $50 \mathrm{~cm}$. Under greater deformations, the interference pattern could not be visually observed.

In Fig. 4a, shown are the photograph of the interferogram and plot of intensity distribution for the case of undisturbed bundle, the fringe contrast is $V_{0}=0.57$. Fig. $4 \mathrm{~b}$ displays the photograph and plot for the case of the disturbed bundle with the bending deflection of $\sim 2 \mathrm{~cm}$, the fringe contrast is $V=0.27$. Extending theoretical consideration to non-ideal interference conditions, i.e., backlight on the screen, different intensity of interfering beams, etc., we can obtain the following expression relating $V, V_{0}$ and $\sigma$ : $\frac{V}{V_{0}}=\exp \left(-\frac{\sigma^{2}}{2}\right)$. With this, one can estimate a value of $\sigma$ and $\rho(\psi)$ for a certain measurement. For the situation depicted in Figs $4 \mathrm{a}, \mathrm{b} \sigma=1.22 \approx 0.4 \pi$ and $\rho(\psi)=\frac{1}{0.4 \pi \sqrt{2 \pi}} \exp \left(-\frac{\psi^{2}}{2(0.4 \pi)^{2}}\right)$. Now a histogram may be built pointing a part of fibers in the bundle having a certain value of the phase shift. In Fig. 5, presented is the data that correspond to the experimental value $\sigma=0.4 \pi$. It is seen that, in this case, $80 \%$ of fibers in the bundle are characterized by the phase shift less than $0.45 \mathrm{rad}$ and only $40 \%$ have the phase shift $0.2 \mathrm{rad}$.

Thereby, the practical result of the work is a proved capability of holographic interferometers with flexible lightguiding bundle under considerable deformation of the bundle. This gives an opportunity to apply such

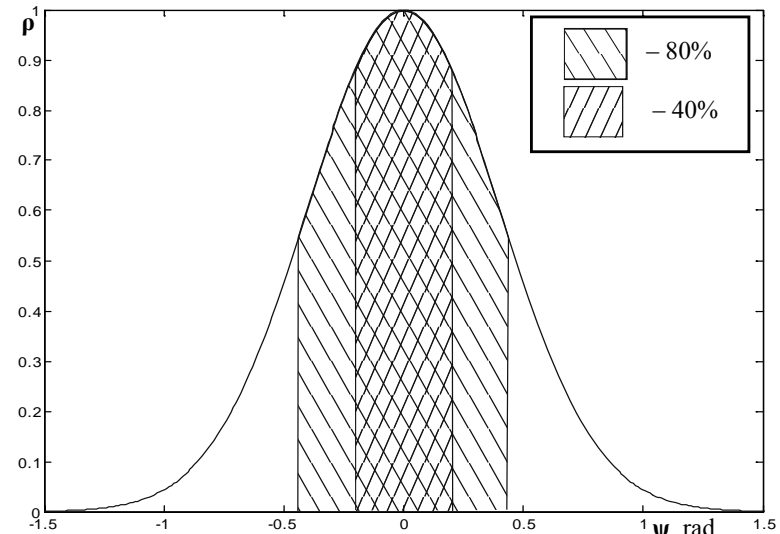

Fig. 5. Statistics of the fibers in the bundle restricted above in magnitude of phase changes (corresponds to the experimental value $\sigma=0.4 \pi$ )

interferometers for acquiring an interferometric information from hardly accessible locations in real (non-laboratory) environment. On the other hand, the obtained results may be useful in the scientific context for investigating a dynamics of various phase structures.

\section{References}

1. Charles M. Vest, Holographic interferometry. Mir, Moscow, 1982, p. 69-77 (in Russian).

2. R. Collier, K. Burkhart, L. Lin, Optical holography. Moscow, Mir, 1973, p. $472-509$ (in Russian).

3. L. Landau, E. Lifshits, Statistical physics. Gos. izdat. tekhno-teoreticheskoy literatury, Moscow Leningrad, 1951, p. 355-362 (in Russian).

4. G. Korn, T. Korn, Mathematic handbook. Nauka, Moscow, 1978, p. 831 (in Russian).

5. O.V. Zolochevkaja, K.A. Gnatovskij, A holographic interferometer on the basis of multimode lightguiding bundles // Proc. SPIE 2648, p. 694697 (1995). 